\title{
VOLUMEN ÓPTIMO DE BALSA EN REDES COLECTIVAS DE RIEGO A LA DEMANDA. CASO DE ESTUDIO CULTIVO DE VIÑA EN ESPAÑA
}

\author{
Izquiel A. ${ }^{1}$; Moreno M. A. ${ }^{2}$; Tarjuelo J. M. ${ }^{3}$; \\ 1 Personal Investigador, Doctorando, Centro Regional de Estudios del Agua (CREA), \\ Universidad de Castilla-La Mancha, Ctra. de Las Peñas km. 3.4, 02071 Albacete, \\ Argenis.Izquiel@alu.uclm.es \\ 2 Profesor Contratado Doctor, Centro Regional de Estudios del Agua (CREA), Universidad \\ de Castilla-La Mancha, Ctra. de Las Peñas km. 3.4, 02071 Albacete, \\ Miguelangel.Moreno@uclm.es \\ 3 Profesor Titular de Universidad, Centro Regional de Estudios del Agua (CREA), \\ Universidad de Castilla-La Mancha, Ctra. de Las Peñas km. 3.4, 02071 Albacete, \\ Jose.Tarjuelo@uclm.es
}

\section{Resumen}

Se ha desarrollado la herramienta DRODN (Design of Reservoir On Demand Network) (implementada en MATLAB) con un enfoque holístico para determinar en una red colectiva de riego a la demanda el mínimo coste total $\left(\mathrm{C}_{\mathrm{T}}\right)$ (incluyendo los costes de inversión $\left(\mathrm{C}_{\mathrm{i}}\right)$, operación $\left(\mathrm{C}_{\mathrm{e}}\right)$ y mantenimiento $\left(\mathrm{C}_{\mathrm{m}}\right)$ ). El procedimiento determina el volumen óptimo de la balsa de almacenamiento y regulación intermedia teniendo en cuenta las tarifas eléctricas y la disponibilidad de agua en el sondeo, la evolución de la demanda de agua de la red a lo largo de la campaña de riegos utilizando el método de curvas aleatorias de demanda diaria (Random daily demand curve) (RDDC). La herramienta se ha aplicado a una red de riego de 171 ha, que riega por goteo viña y olivar, localizada en la provincia de Cuenca (España). Los resultados indican que el volumen óptimo de almacenamiento y regulación está alrededor de los $5000 \mathrm{~m}^{3}$, y el $\mathrm{C}_{\mathrm{T}}$ para $\mathrm{ND}=100 \mathrm{~m}$ es $325 €$ ha $^{-1}$ año- ${ }^{-1}$. El $\mathrm{C}_{\mathrm{e}}$ es el principal componente del $\mathrm{C}_{\mathrm{T}}$, representando más del $63 \%$ en los casos estudiados. La forma de operación y manejo de la red de riego condiciona las dimensiones de la balsa y los costes anuales de suministro.

\section{1- Introducción y Objetivos}

En países como España, donde el coste de la energía eléctrica está experimentando un gran incremento (más de un 100\% desde 2008), unido a la gran dependencia energética de sus regadíos al tener más del $70 \%$ de los 3,4 millones de hectáreas regadas con riego a presión ( $48 \%$ de riego localizado y $22 \%$ de aspersión) (ESYRCE, 2011), y a la cada vez menor disponibilidad de agua para riego por incremento de las necesidades urbanas y medioambientales, resulta imprescindible desarrollar herramientas y modelos que ayuden a mejorar la eficiencia en el uso del agua y la energía en el regadío (Pulido-Calvo y GutiérrezEstrada, 2011; Moreno et al., 2012). Las balsas de almacenamiento y regulación, situadas entre la fuente de agua y el sistema de riego, es una opción que permite regular el caudal bombeado y el momento de consumo de energía, buscando reducir los costes energéticos (Nel y Haarhoff, 1996; Hirose, 1997). La procedencia del agua utilizada para riego en regiones como la Mancha Oriental es $92 \%$ de sondeos, $6 \%$ de ríos o manantiales y $2 \%$ 
mixtos (JCRMO, 2012); ésa necesidad de extraer agua a profundidades entre 50 y $200 \mathrm{~m}$, obliga a disponer de herramientas de ayuda a la toma de decisiones para el adecuado manejo del agua y de la energía. El objetivo de este trabajo es desarrollar un modelo de optimización denominado DRODN (Design of regulating Reservoire On-Demand irrigation Networks) con un enfoque holístico para encontrar el mínimo coste $\left(\mathrm{C}_{\mathrm{T}}\right)$ de entrega de agua a la cabecera de una red de riego a la demanda, teniendo en cuenta la variabilidad de presión y caudal en la red durante la campaña de riegos. El modelo contempla la utilización de una balsa intermedia para buscar el mínimo $\mathrm{C}_{\mathrm{T}}$, incluyendo la inversión, la energía y el mantenimiento necesarios en el proceso de extracción y entrega de agua en cabecera de la red de riego. El trabajo analiza el nivel dinámico de bombeo (ND) sobre el $\mathrm{C}_{\mathrm{T}}$, determinando los caudales óptimos de bombeo y rebombeo, y la capacidad de la balsa que conducen al $\mathrm{C}_{\mathrm{T}}$ mínimo. La herramienta se ha aplicado a una red de riego por goteo, y con agua procedente del acuífero Mancha Oriental, en la provincia de Cuenca (España).

\section{2- Materiales y Métodos}

\section{1- Optimización de la alimentación a una red de riego a la demanda}

Considerando el proceso de extracción y suministro de agua a una red colectiva en su conjunto, se ha desarrollado el modelo DRODN (Figura 1) con un enfoque holístico para minimiza el coste total anual de aplicación del agua de riego $\left(\mathrm{C}_{\mathrm{T}}\right.$, en $€ \mathrm{ha}^{-1}$ año $\left.^{-1}\right)$, como suma de los costes de inversión $\left(C_{i}\right)$, energía $\left(C_{e}\right)$ y mantenimiento $\left(C_{m}\right)$. Los costes de inversión incluyen el sondeo, la línea eléctrica, las bombas, la red de tuberías en la impulsión y distribución hasta la cabecera de la red.

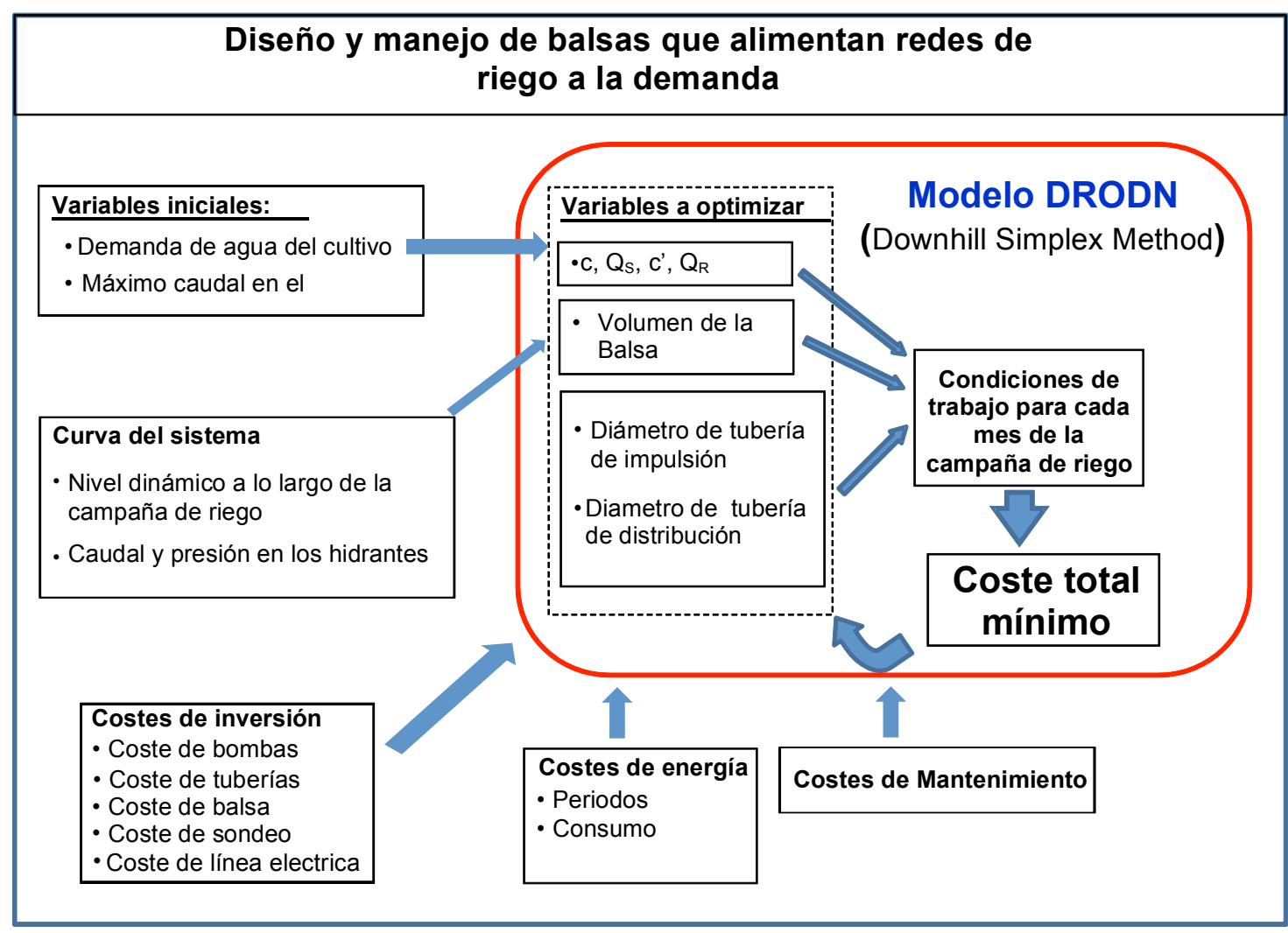

Figure 1. Esquema del proceso de optimización para minimizar los costes totales de aplicación de agua $\left(\mathrm{C}_{\mathrm{T}}\right)$ en redes de riego a la demanda. 
Las variables a optimizar fueron: a) el caudal bombeado desde el sondeo a la balsa $\left(Q_{S}\right)$ y el de rebombeo desde la balsa hasta la red de riego $\left(Q_{R}\right)$; b) los coeficientes de la curva característica de la bomba en el sondeo (c) y en el rebombeo (c') que determinan la forma óptima; c) los diámetros de las tuberías de impulsión $\left(D_{i}\right)$ y de las tuberías de distribución $\left(\mathrm{D}_{d}\right)$; y d) las dimensiones de la balsa de regulación de acuerdo con el volumen óptimo de la balsa $\left(\mathrm{V}_{\mathrm{T}}\right)$. El proceso de optimización se llevó a cabo mediante el método Downhill Simplex Method (Nelder y Mead, 1965) con el objetivo de minimizar los costes totales (ecuación 1):

$$
\mathrm{MIN} \mathrm{C}_{\mathrm{T}}=C_{i}+C_{e}+C_{m}
$$

donde $C_{i}=$ coste anual de inversión, $C_{e}=$ coste anuales de operación, el cual incluye los costes energéticos y $\mathrm{C}_{m}=$ coste anual de mantenimiento.

Los datos que alimentan el modelo son: el consumo de agua de los cultivos $\left(N_{n}\right)$, el nivel dinámico (ND) del agua en el sondeo, la presión necesaria a la entrada de la red $\left(\mathrm{P}_{\mathrm{o}}\right)$, máximo caudal a extraer del sondeo $\left(Q_{0}\right)$, los precios de la energía eléctrica $(\mathrm{Pe})$, los precios de los equipos y elementos de las instalaciones hasta la cabecera de la red de riego.

\section{2- Descripción del caso de aplicación del modelo}

El estudio se llevó a cabo en una red de riego por goteo a la demanda de 171 ha para riego de viña $(80 \%)$ y olivar $(20 \%)$, localizada en la Grajuela, provincia de Cuenca (España). El agua procede de un sondeo de $135 \mathrm{~m}$ de profundidad, nivel dinámico de 110 $\mathrm{m}$, y es extraída por una bomba de $296 \mathrm{HP}$, con un caudal teórico de $130 \mathrm{I} \mathrm{s}^{-1}$, y almacenada en una balsa de $20000 \mathrm{~m}^{3}$ (de tronco piramidal invertida) desde donde se rebombea a la red de riego. Las bombas son controladas por un PCL (controlador lógico programable), para mantener una presión en la cabecera de la red de $45 \mathrm{~m}$ y garantizar una presión en hidrante de $25 \mathrm{~m}$.

\section{3- Métodos para estimar la demanda de agua de la red}

Para obtener el volumen de la balsa que regule la máxima demanda de agua de los cultivos durante los 10 días del periodo de consumo punta la optimización se realiza por etapas. En una primera etapa se aplica el DRODN para obtener una aproximación del volumen útil de balsa $\left(\mathrm{V}_{\mathrm{u}}\right)$ y el tiempo de operación óptimo en la red (OT) y en el sondeo $\left(\mathrm{OT}_{\mathrm{s}}\right)$ a partir de las necesidades medias mensual de agua de los cultivos $\left(L_{m}\right)$ para satisfacer las necesidades máximas de caudal y altura. En una segunda etapa con el valor de OT se calcula el caudal demandado mediante el algoritmo de curvas aleatorias de demanda diaria (RDDC) (Moreno et al., 2007) y se procede a calcular con la herramienta DRODN el volumen óptimo de balsa, según se describen a continuación:

1.- La primera etapa consiste en cuantificar la demanda media diaria de agua en la red $\left(L S^{-1}\right)$ en función de la eficiencia de riego $\left(E_{a}\right)$, la superficie a regar $(S)$, la evapotranspiración de la alternativa de cultivos (ETc) (calculada por la ecuación de Penman Monteith), y las pérdidas producidas en la balsa $\left(P_{b}\right)$, que se estiman en un $7 \%$ del agua almacenada (Molina, 2004). La distribución de la demanda mensual para la alternativa de cultivos fue de 19,$7 ; 38,3 ; 65,0 ; 49,9$ y $3,1 \mathrm{~mm}$ regando de mayo a septiembre respectivamente, lo que supone $176 \mathrm{~mm}^{2} \mathrm{n}^{-1}$.

2.- La segunda etapa consiste en obtener la distribución de los caudales de una red de riego a la demanda a partir de la generación diaria de caudales aleatorios (RDDC) considerando la apertura aleatoria de hidrantes, el tiempo de operación (OT), los parámetros de riego (intervalo $\left(I_{r}\right)$ y tiempo de riego $\left(T_{r}\right)$ ) de cada parcela y tipo de cultivo, y generando una curva aleatoria de demanda al sumar la descarga de todos los hidrantes abiertos localizados aguas abajo de la red. Este caudal se obtuvo cada 15 min según el OT 
(obtenidos en una primera etapa con $L_{m}$ ) para los 10 días de máxima necesidades de agua del cultivo; todo el cálculo de demanda se hizo a fin de evaluar la capacidad de regulación de la balsa y posteriormente calcular el volumen de la balsa con DRODN considerando el caudal máximo $\left(Q_{\max }\right)$ obtenido con la metodología RDDC; para el resto de los días de la campaña de riego la demanda se cuantificó con la metodología de $L_{m}$ de la primera fase.

Se evaluaron los costes anuales para garantizar el caudal y la presión en la cabecera de la red de riego, así como el tamaño de balsa de regulación, contemplando 9 escenarios de niveles dinámicos $(0,10,50,75,100,125,150,200$ y 250 m).

\subsection{Diseño de la balsa}

El volumen de la balsa se calculó considerando la demanda de agua de la alternativa de cultivos $\left(\mathrm{N}_{\mathrm{n}}\right)$, la disponibilidad de agua en el acuífero, las horas de bombeo utilizadas y las curvas características del sistema de bombeo.

Se consideró una balsa con forma tronco piramidal invertido de base cuadrada, semienterrada, cubierta con lámina de polietileno (Figura 2). El volumen de la balsa $\left(V_{T}\right)$ es el volumen útil $\left(\mathrm{V}_{\mathrm{u}}\right)$ incrementado en $20 \%$ como margen de seguridad (Jowitt y Germanopoulo 1992, Amigó y Aguiar 1994). Para el cálculo del $C_{T}$ se consideró además que $V_{u}$ no fuera inferior a la mayor demanda de agua diaria en todo el período de funcionamiento del sistema (Pulido-Calvo et al., 2003).

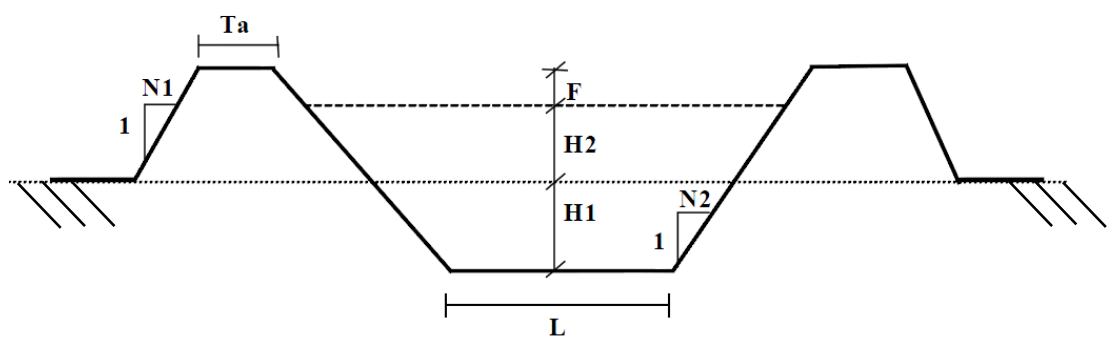

Figura 2. Sección transversal de la balsa de regulación y variables que la describen.

Para establecer las dimensiones geométricas ( $\mathrm{L}, \mathrm{H} 1$ y $\mathrm{H} 2)$ que hacen mínimo el coste de inversión de la balsa para un $\mathrm{V}_{\mathrm{T}}$ conocido, se desarrolló un algoritmo específico de cálculo usando el método numérico de newton (Conte y Boor, 1974); se fijaron los valores de $\mathrm{N} 1=1,5 ; \mathrm{N} 2=2 ; \mathrm{Ta}=5 \mathrm{~m} ; \mathrm{F}=1 \mathrm{~m} ; \mathrm{y} \mathrm{CF}=10 \%$ (CF es el coeficiente para compensar la diferencia de volumen en el movimiento de tierra debido al nivel de compactación) que dependen de los métodos y técnicas de construcción (Amigó y Aguiar 1994, Pulido-Calvo et al., 2006 y CNEGP, 2010). Las alturas del agua contenida en el depósito $(\mathrm{H} 1+\mathrm{H} 2)$ se consideraron en el rango de 2 a 12 m (Giscosa, 1995).

Una vez calculado el $V_{u}$ se realiza un segundo cálculo que indique el volumen mínimo de regulación $\left(\mathrm{V}_{\min }\right)$, desarrollando un algoritmo basado en el concepto de periodo de vaciado (López et al., 1993), partiendo de la demanda diaria de agua de la red en periodo punta y las necesidades de rebombeo a la red de riego, el aporte potencial horario del bombeo $(\mathrm{APH})$ del sistema de bombeo y las horas optimizadas para el llenado $\left(\mathrm{H}_{\mathrm{Ui}}\right)$ y vaciado de la balsa $\left(\mathrm{H}_{\cup d}\right)$ teniendo en cuenta los costes de la energía en los distintos periodos tarifarios (Figura 3). Con este procedimiento se obtienen los balances de volumen en la balsa cada 15 min durante los 10 días críticos del mes de máxima demanda del cultivo y se obtiene el $V_{\min }$. 


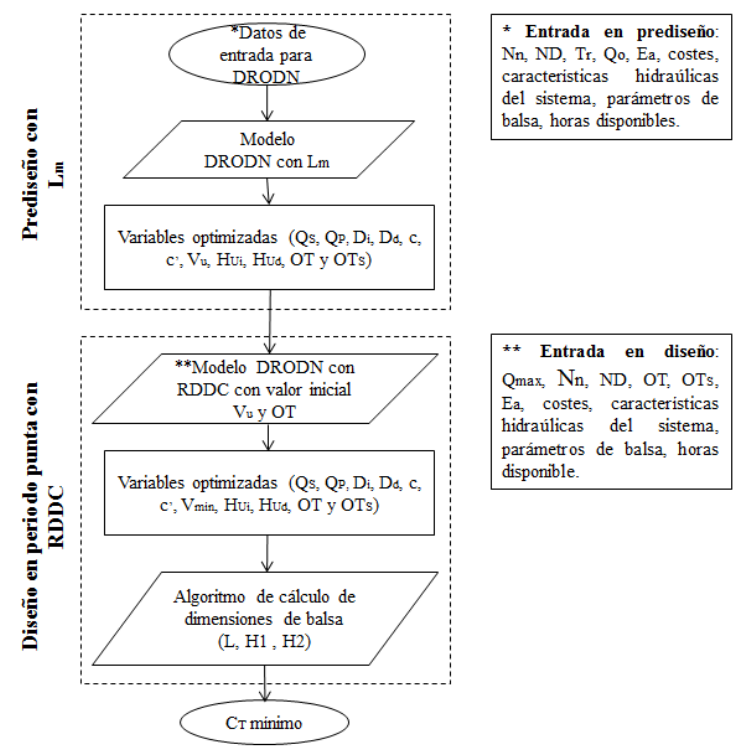

Figura 3.- Organigrama de cálculo de volumen mínimo de regulación en balsa.

\section{5- Costes anuales de inversión}

A fin de determinar los costes anuales de inversión desde sondeo hasta la entrega de agua en cabecera de la red de riego $\left(C_{a}\right.$, en $€ \cdot$ ha $^{-1}$.año $\left.{ }^{-1}\right)$, se consideraron los precios medios de diferentes empresas de manufactura, distribución y servicios de riego en España. Los componentes de los costes de bombas, tuberías e instalación en bombeo se agruparon en ecuaciones de regresión, los coeficientes de determinación de las curvas de regresión de costes fueron altamente significativos $(>0,94)$.

La anualidad de la inversión ( $A=C R F . C_{i}$, en $€ a n o^{-1}$ ) correspondiente a los costes de inversión $\left(\mathrm{C}_{\mathrm{i}}\right.$, en $€$ ) se calculó considerando una vida útil $(\mathrm{n})$ de 12 años para bombas y 24 años para el resto de equipos, tubería y accesorios (Scherer y Weigel, 1993), y una tasa de interés (i) de 6\%. El factor de recuperación del capital (CRF) y la anualidad de la inversión por unidad de superficie $\left(\mathrm{C}_{\mathrm{a}}\right.$, en $\left.€ \mathrm{ha}^{-1} \mathrm{año}^{-1}\right)$ fueron calculados mediante las ecuaciones (4) y (5).

$$
\begin{aligned}
& C R F=\frac{i(1+i)^{n}}{(1+i)^{n}-1} \\
& C_{a}=\frac{A}{S}=\frac{C R F \cdot C_{i}}{S}
\end{aligned}
$$

donde $\mathrm{S}$ es el área regada por la red colectiva de riego (ha).

\section{6- Costes anuales de operación}

La potencia absorbida ( $N_{p}$, en $\mathrm{kW}$ ) en la extracción de agua desde el sondeo y en la alimentación a red de riego para las condiciones de funcionamiento de cada mes de la campaña de riego, fueron calculadas según la presión $\left(H_{T}\right.$, en $\left.\mathrm{m}\right)$ y el caudal $\left(Q\right.$, en $\left.\mathrm{m}^{3} \mathrm{~s}^{-1}\right)$ necesarios para el adecuado funcionamiento del sistema mediante la ecuación (6).

$$
N_{p}=\frac{9,81 Q H_{T}}{\eta}
$$


donde $\eta=$ es la eficiencia del sistema de bombeo (0,6 en caso de estudio). Las pérdidas de carga por fricción en tubería fueron calculadas con la ecuación de Hazen-William. En las tuberías las pérdidas locales han sido estimadas en un $10 \%$ de las pérdidas por fricción.

Los costes operacionales anuales por unidad de área $\left(C_{e}, € \cdot\right.$ ha $^{-1} \cdot$ años $\left.^{-1}\right)$ se calcularon dividiendo los $C_{o p}$ por el área regada ( $\mathrm{S}$, en ha), siendo $C_{o p}$ la suma de los costes anuales en el sondeo y en la inyección de agua a la red. En cada caso fueron calculados con las ecuaciones 7 y 8 .

$$
\begin{aligned}
& \mathrm{C}_{\mathrm{op}}=\text { Acceso a potencia }+ \text { Consumo energético } \\
& \mathrm{C}_{\mathrm{op}}=\sum_{\mathrm{i}=1}^{12} \sum_{\mathrm{j}=1}^{\mathrm{k}}\left(\mathrm{N}_{\mathrm{p}}\right)_{\mathrm{i}} \mathrm{Pa}_{\mathrm{ij}}+\sum_{\mathrm{i}=1}^{12} \sum_{\mathrm{j}=1}^{\mathrm{k}}\left(\mathrm{N}_{\mathrm{p}}\right)_{\mathrm{i}} \mathrm{T}_{\mathrm{ij}} \mathrm{P}_{i j}
\end{aligned}
$$

donde $N_{p}=$ potencia de la bomba calculada a partir de la ecuación (6) en kW; T = tiempo de operación mensual de la bomba en horas; $\mathrm{Pa}=$ precio de acceso a la potencia $\left(€ \mathrm{~kW}^{-1} \mathrm{mes}^{-}\right.$ $\left.{ }^{1}\right) ; P=$ precio de la energía en $€ . \mathrm{kW}^{-1} \mathrm{~h}^{-1}$; los índices $i$ y $j$ se refiere a los meses y precio de la energía en las horas $(k)$ durante el día, respectivamente.

Los costes energéticos fueron los vigentes en España para 2012 publicados por el Boletín Oficial del Estado (BOE). Se consideró la posibilidad del cambio de precio de la energía (EAE) debido a la tasa de inflación, por lo que se usa la ecuación de ajuste de Keller y Bliesner (1990).

\section{7- Costes de mantenimiento}

Se adicionó un $5 \%$ de los costes de inversión, como costes de mantenimiento necesario para que el sistema planteado cumpla con su vida útil de 12 años para bombas, y 24 años para el resto de equipos, tuberías y accesorios.

\section{8- Costes de extracción del agua $\left(\mathrm{C}_{\mathrm{S}}\right)$ y coste de alimentación a la red de riego $\left(C_{R}\right)$}

Dentro del $\mathrm{C}_{\mathrm{T}}$ se puede distinguir a su vez entre el coste de extracción del agua $\left(\mathrm{C}_{\mathrm{S}}\right)$ y el coste de entrega del agua a la cabecera de la red de riego $\left(C_{R}\right)$, siendo el $C_{T}$ la suma de los dos. El $\mathrm{C}_{S}$ es el de transportar el agua desde la fuente hasta la a balsa incluyendo los costes de inversión $\left(\mathrm{C}_{\mathrm{aS}}\right)$ y de operación $\left(\mathrm{C}_{\mathrm{eS}}\right)$ en este proceso, dejando el agua a presión atmosférica a la salida. El $\mathrm{C}_{\mathrm{R}}$ es el de llevar el agua desde la balsa hasta la cabecera de la red de riego, con las condiciones de presión y caudal que necesite en cada momento.

Los coste anual de extracción del agua $\left(\mathrm{C}_{S} ; € \mathrm{ha}^{-1}\right.$ año $\left.^{-1}\right)$, y de entrega de agua a la red $\left(C_{R} ; €\right.$ ha $^{-1}$ año $\left.{ }^{-1}\right)$ (ecuaciones 8 y 9 ) serán, es la suma de los de inversión, incluido el $5 \%$ para mantenimiento, y de energía.

$$
\begin{aligned}
& C_{S}=\left(C_{a S}+C_{e S}\right) \\
& C_{R}=\left(C_{a R}+C_{e R}\right)
\end{aligned}
$$

donde $\mathrm{C}_{\mathrm{as}}$ y $\mathrm{C}_{\mathrm{aR}}=$ los costes anuales de inversión, incluido el mantenimiento, $\left(€ \mathrm{ha}^{-1} \mathrm{año}^{-1}\right)$ para la extracción y suministro de agua a la red; $\mathrm{C}_{\mathrm{es}}$ y $\mathrm{C}_{\mathrm{eR}}=$ los costes anuales de energía $\left(€\right.$ ha $^{-1}$ año $^{-1}$ ) para la extracción y suministro de agua a la red. Estos mismos costes se pueden expresar también por unidad de volumen bombeado (ecuaciones 10 y 11).

$$
\begin{aligned}
& C_{w S}=\left(C_{a S}+C_{e S}\right)^{*} N_{n}^{-1 *} E_{a} \\
& C_{w R}=\left(C_{a R}+C_{e R}\right)^{*} N_{n}{ }^{-1 *} E_{a}
\end{aligned}
$$

donde $\mathrm{C}_{\mathrm{ws}}$ y $\mathrm{C}_{\mathrm{wR}}=$ los costes anuales de extracción del agua y de entrega de agua a la red respectivamente $\left(€ \mathrm{~m}^{-3}\right.$ año $\left.{ }^{-1}\right) ; N_{n}=$ Necesidades netas de riego $\left(\mathrm{m}^{3} \mathrm{ha}^{-1}\right.$ año $\left.{ }^{-1}\right) ; E_{a}=$ 
eficiencia general de aplicación del agua por la red de riego (Keller y Bliesner, 1990). En este trabajo se consideró $\mathrm{E}_{\mathrm{a}}=88 \%$ (Carrión et al., 2013).

\section{3- Resultados y Discusión}

\section{1- Volumen óptimo de balsa}

Al calcular el volumen útil de la balsa $\left(V_{u}\right)$ para garantizar el agua consumida en el día de mayor consumo o el volumen mínimo necesario para la regulación $\left(\mathrm{V}_{\min }\right)$, resultó que, $V_{u}$ fue hasta un $73 \%$ mayor que el $V_{\text {min }}$ para $N D<50 \mathrm{~m}$ y ND> $150 \mathrm{~m}$, y un $71 \%$ mayor para ND entre 50 y 150 m (Figura 4). Los menores valores de $V_{\min }$ se obtienen cuando las horas de operación de la red son muy similares a los tiempos de operación en sondeo. En cambio cuando las horas de funcionamiento de la red son mayores que las de extracción de agua resultan volúmenes de regulación mayores, al resultar mayor el caudal óptimo de la extracción de agua

La balsa en la red de riego de la zona de estudio tiene un $V_{T}$ de $20000 \mathrm{~m}^{3}$ y fue diseñada para un posible cambio de cultivos de mayor demanda de agua y una posible ampliación de la zona regada, por lo que actualmente este volumen resulta excesivo considerando lo estable del suministro de agua por el bombeo. El optimizador indica un $\mathrm{V}_{\mathrm{T}}$ medio de la balsa entorno a los $5000 \mathrm{~m}^{3}$, es decir $75 \%$ inferior al volumen diseñado. Reca et al. (2014) señalan que el aumento de los costes energéticos favorece el uso de balsas en cualquier sistema de riego ya que permite reducir los costes, principalmente en las condiciones semiáridas españolas, por lo que el tamaño resultante es bastante sensible a las variaciones del precio de la energía.

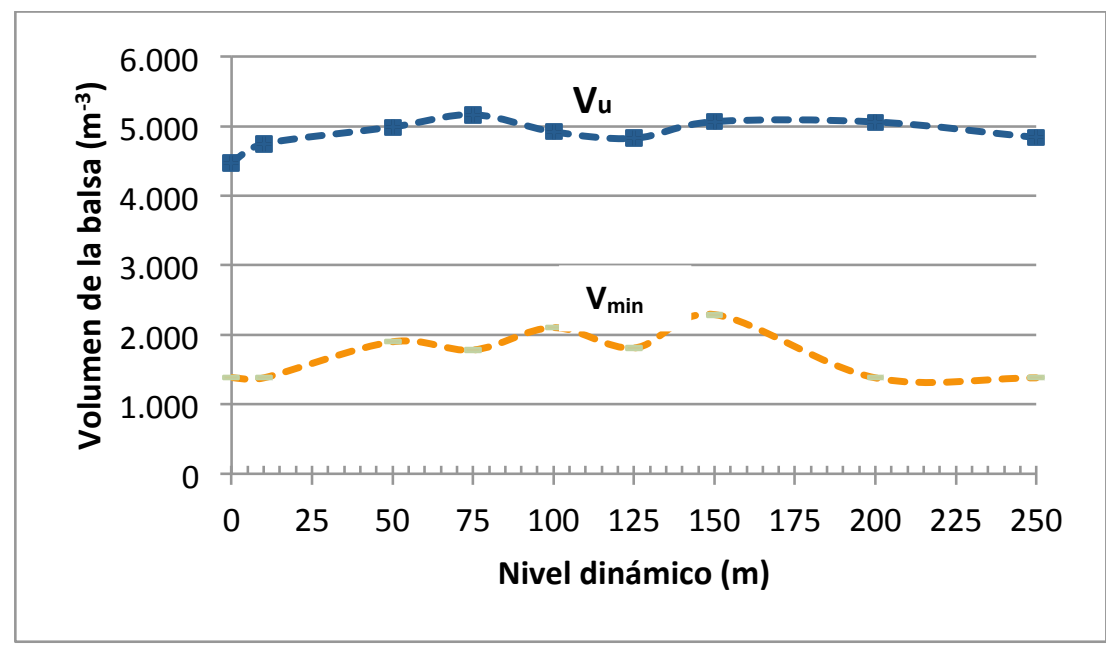

Figura 4- Volumen de balsa, útil $\left(\mathrm{V}_{\mathrm{u}}\right)$ y mínimo $\left(\mathrm{V}_{\mathrm{min}}\right)$ en la red de riego.

La metodología de RDDC por su condición probabilística genera las ligeras oscilaciones de los volúmenes de balsa que se aprecian en la Fig. 4. Los menores volúmenes de regulación $\left(\mathrm{V}_{\min }\right)$ obtenidos se deben a que usan mayor número de horas de bombeo (Figura 8).

\section{2- Coste total $\left(C_{T}\right)$ de suministro de agua a una en red de riego a la demanda.}

El $C_{T}$ crece directamente proporcional con el ND (Figura 5), su coeficiente de determinación $\left(R^{2}\right)$ es $>0,98$. La principal componente del $C_{T}$ es la energía $\left(C_{e}\right)$ (entre 57 y $78 \%$ de los $\mathrm{C}_{\mathrm{T}}$ ). Los $\mathrm{C}_{\mathrm{e}}$ son los que originan el crecimiento sostenido del $\mathrm{C}_{\mathrm{T}}$ ya que los $\mathrm{C}_{\mathrm{i}}$ experimentan un crecimiento pequeño hasta ND $=150 \mathrm{~m}$, manteniéndose en valores 
similares para ND superiores. Esto último es debido a que la solución óptima elige bombas de menor potencia $\left(<C_{a}\right)$ pero con mayor número de horas de funcionamiento $\left(>C_{e}\right)$.

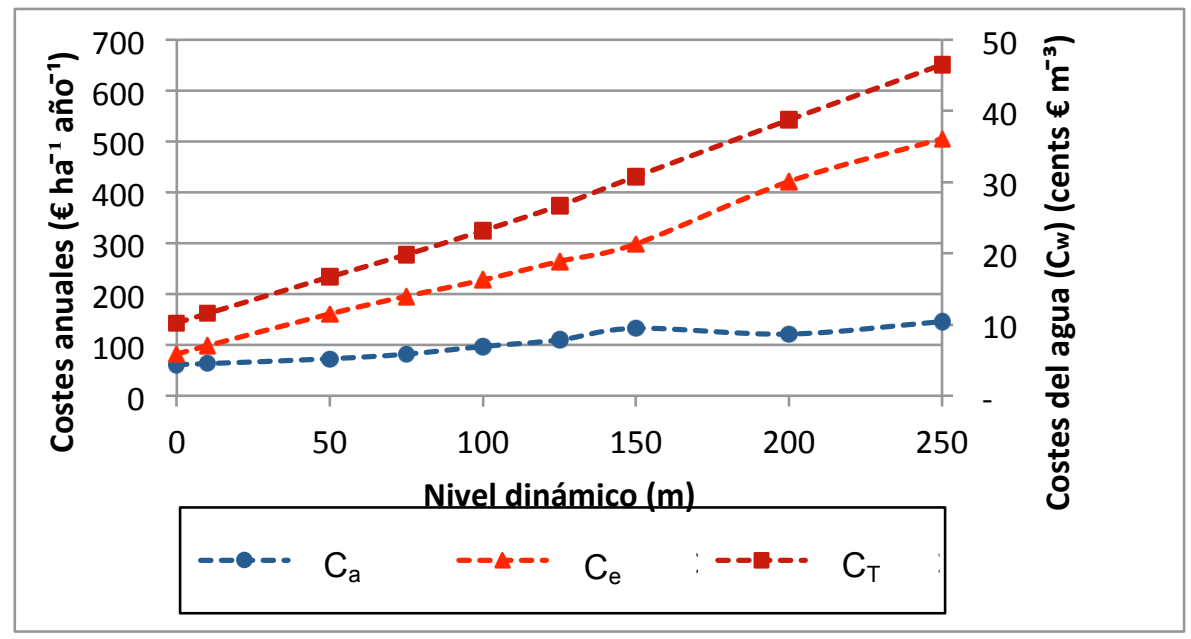

Figura 5- Coste total $\left(C_{T} €\right.$ ha ${ }^{-1}$ año $\left.{ }^{-1}\right)$, costes de inversión $\left(C_{a}\right)$, costes energéticos $\left(C_{e}\right)$ y costes de entrega del agua a la red de riego $\left(C_{w}\right)$.

\section{3- Costes del metro cúbico de agua entregada a la red de riego $\left(C_{w}\right)$.}

Los resultados se muestran en la Figura 5. Los $C_{w e}$ están por encima de los $C_{w a}$ en todos ND y los escenarios considerados, llama la atención el incremento del coste de energía en la extracción al aumentar el ND creciendo a razón de 0,11 cent $€ \mathrm{~m}^{-3} \mathrm{~m}^{-1}$, mientras que los costes de inversión crecen en menor medida $\left(0,02\right.$ cent $\left.€ \mathrm{~m}^{-3} \mathrm{~m}^{-1}\right)$.

Los costes totales de entrega de agua a la red dependen de la profundidad de extracción de agua, aumentando desde 9,6 hasta 43,4 cent $€ \mathrm{~m}^{-3}$ para ND $=0$ y $250 \mathrm{~m}$ respectivamente (Figura 5). Los $\mathrm{C}_{\mathrm{w}}$ para ND $=100 \mathrm{~m}$, están en torno a 21 cent $€ \mathrm{~m}^{-3}$, esto valores siguen siendo elevados para las explotaciones agrícolas de la Mancha Oriental, en Albacete, que admitirían un máximo de 18 cent $€ \mathrm{~m}^{-3}$ (Castillo, 1997).

\section{4- Costes de extracción de agua $\left(C_{S}\right)$ y del rebombeo a la red de riego $\left(C_{R}\right)$.}

En la Figura 6 se recoge, la evolución de los costes de energía (Fig. 6a) y de inversión (Fig. 6b) con el ND. El comportamiento del $\mathrm{C}_{\mathrm{T}}$ antes comentado viene originado por el proceso de extracción del agua ya que en el rebombeo para alimentar a la red, tanto los costes anuales de inversión $\left(C_{a R}\right)$ como de energía $\left(C_{e R}\right)$ experimentan escasas variaciones $\left(<16 € \mathrm{ha}^{-1} \mathrm{año}^{-1}\right)$ con el ND al tener que suministras una presión en cabecera de red constante ( $45 \mathrm{~m}$ en este estudio) y la escasa variación del nivel de agua en la balsa.

Las diferencias de comportamiento de la solución óptima de mínimo $\mathrm{C}_{\mathrm{T}}$, se manifiestan principalmente en la extracción. Se produce un incremento del $\mathrm{C}_{\mathrm{es}}$ en el proceso de extracción de agua para ND > $150 \mathrm{~m}$ (Fig. 6a) debido a que el optimizador selecciona menor caudal (Fig. 7b), con menor potencia (Fig. 7a), por lo que requiere mayor número de horas de funcionamiento (Fig 6a) (> $\mathrm{C}_{e s}$ ). Esta es una fortaleza de la herramienta DRODN, al poder estudiar el proceso de extracción y suministro de agua a la red en su conjunto, teniendo en cuenta la variación de la demanda de agua durante la campaña de riegos. 

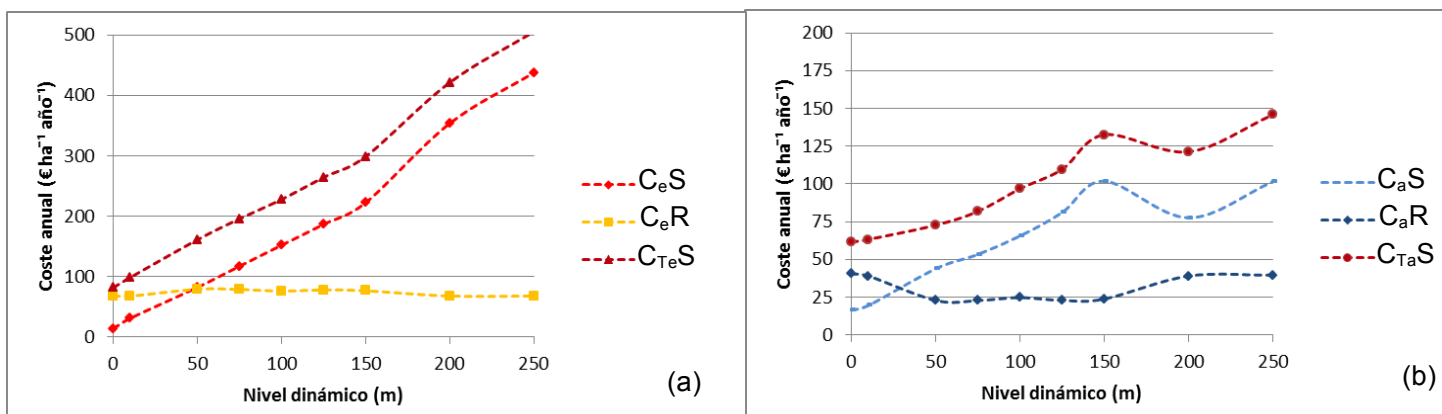

Figura 6- Coste anuales total $\left(C_{T} €\right.$ ha $^{-1}$ año $\left.{ }^{-1}\right)$, desglosados: a) costes energéticos $\left(C_{e}\right)$ y b) costes de inversión $\left(C_{a}\right)$, en la extracción de agua $(S)$ y en rebombeo a la red de riego $(R)$.

\section{5- Relación entre el caudal y el consumo de energía en los distintos periodos tarifarios.}

En las Figuras 7 y 8 se presenta la evolución de la potencia de bombeo que conduce al $\mathrm{C}_{\mathrm{T}}$ mínimo, del caudal óptimo y el consumo de energía en los distintos periodos tarifarios respectivamente.

Los caudales óptimos de alimentación a la red son algo más de $115 \mathrm{~L} \mathrm{~s}^{-1}$ para ND entre 50 y $150 \mathrm{~m}$ y $278 \mathrm{~L} \mathrm{~s}^{-1}$ para ND $<50 \mathrm{~m}$ y ND > $150 \mathrm{~m}$, y las potencias entre 70 y 170 $\mathrm{kW}$ respectivamente (en los intervalos de ND indicados)(Figura 7), utilizando las horas valle (en torno a $750 \mathrm{~h}$ ) y algunas pocas llano para ND entre 50 y $150 \mathrm{~m}$, y solo una pocas horas valle (en torno a $340 \mathrm{~h}$ ) para ND < $50 \mathrm{~m}$ y ND > 150m (Figura 8).

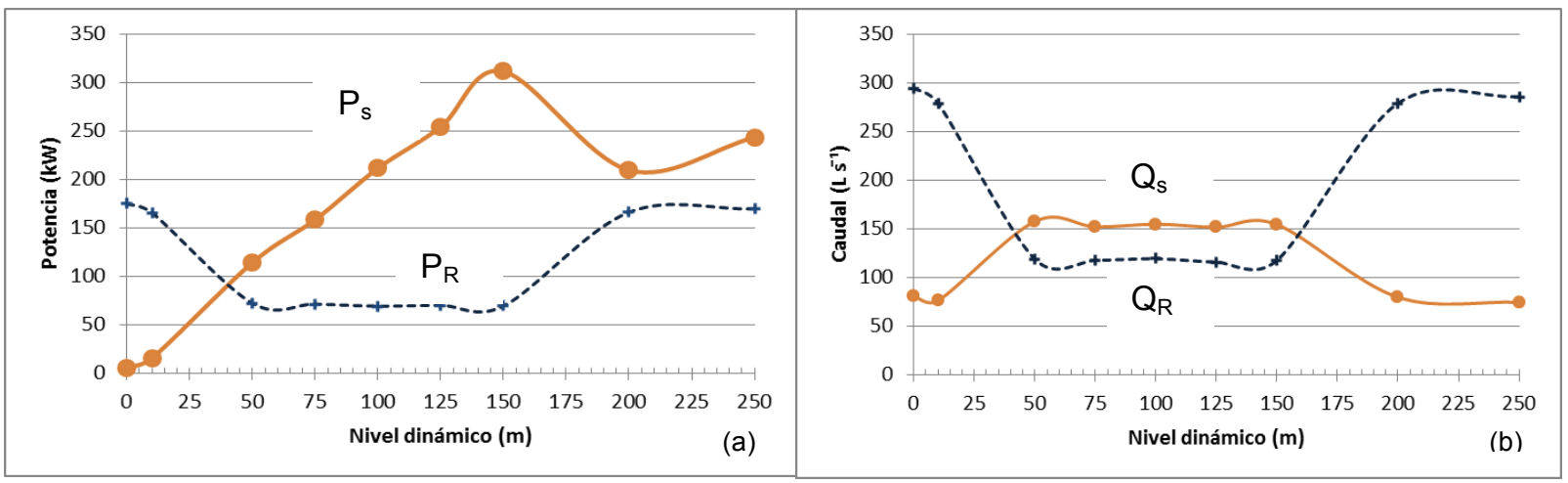

Figura 7- Potencia de bomba consumida en la extracción de agua $\left(P_{S}\right)$ y en el rebombeo a la red de riego $\left(P_{R}\right)$, caudal en la extracción $\left(Q_{S}\right)$ y en la red $\left(Q_{R}\right)$.

En la extracción de agua se produce un cambio de comportamiento de la potencia a partir de ND > $150 \mathrm{~m}$ (Fig. 7a), donde la solución de $\mathrm{C}_{\mathrm{T}}$ mínimo se consigue aumentando el consumo de energía (mayor número de horas valle y llano, (Fig. 8a) y disminuyendo la inversión (Fig. 6b). La razón está en que el coste de inversión en bombas crece exponencialmente con su potencia, por lo que, a partir de un determinado valor de potencia, se obtiene mínimo $\mathrm{C}_{\mathrm{T}}$ aumentando el consumo de energía y disminuyendo la inversión en bombas. Esta es una ventaja de disponer de una herramienta de ayuda en la toma de decisiones como DRODN, al permitir identificar el cambio en la estrategia de optimización del $\mathrm{C}_{\mathrm{T}}$ en función de los costes de inversión y de energía en cada país, pudiendo obtenerse soluciones muy distintas para los mismos sistemas de distribución del agua de riego según sea la relación entre los costes de inversión y de energía. 


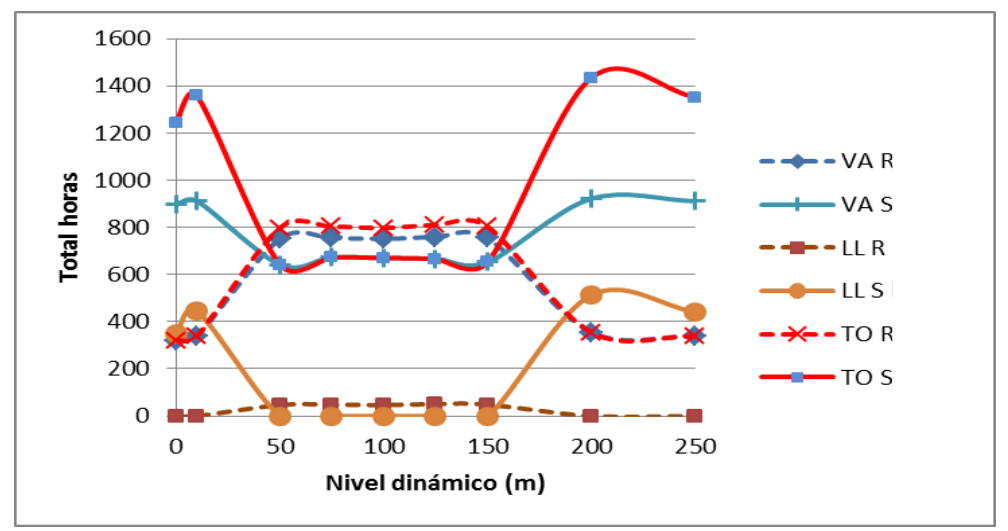

Figura 8- Horas consumidas en valle (VA), llano (LL) y total (TO), en la extracción de agua $(S)$ y el rebombeo a la red de riego $(R)$.

\section{4- Conclusiones y Recomendaciones}

Se ha desarrollado el modelo DRODN bajo el entorno MATLAB como herramienta para optimizar el diseño y la gestión del sistema de distribución de agua, y el almacenamiento para alimentar redes de riego a la demanda, contribuyendo a mejorar la eficiencia del uso del agua y la energía en el regadío. Esta herramienta ayuda en la toma de decisiones en los procesos de modernización de regadíos, donde la escasez de agua para riego y el alto coste de la energía pueden condicionar la viabilidad de esos regadíos.

Dados los altos costes de construcción de balsa, para el cálculo de su tamaño óptimo se debe tener en cuenta la forma de operación y manejo de la red de distribución de agua a fin de abaratar los costes anuales de riego y/o dar mayor garantía de suministro de agua a la red de riego.

El coste energético, es el componente más importante en los $\mathrm{C}_{\mathrm{T}}$ para todos los ND evaluados, pasando de ser $63 \%$ para ND $=0$ a $80 \%$ para ND $=250 \mathrm{~m}$. Por lo que está variable en los costes de riego debe ser muy analizada al momento de poner en marcha cualquier proyecto de riego.

\section{5- Bibliografía}

Amigó E. \& Aguiar, E. (1994). Manual para el diseño, construcción y explotación de embalses impermeabilizados con geomembranas, Dirección General de Estructuras Agrarias, Consejería de Agricultura y Alimentación, Gobierno de Canarias.

Carrión F., Tarjuelo, J.M., Carrión, P., \& Moreno, M.A. (2013). Low-cost microirrigation system supplied by groundwater: An application to pepper and vineyard crops in Spain. Agricultural Water Management, 127, 107-118.

CNEGP. (2010). Manual para el diseño, construcción, explotación y mantenimiento de balsas, Ministerio de medio ambiente y medio rural y marino. Madrid, España: Comité Nacional Español de Grandes Presas. 272 p.

Conte, S. D. \& Boor, C. (1974). La solución de ecuaciones no lineales. Análisis numérico elemental, un enfoque algorítmico. (2da ed.). México: McGraw-Hill.

ESYRCE. (2011). Encuesta de Superficies y Rendimientos de Cultivos en España Ministerio de Medioambiente y Medio Rural y Marino (MARMA). 166 p. 
Giscosa. (1995) Todo sobre la impermeabilización de embalses con geomembranas sintéticas. Impermeabilización profesional, 7: 61-78.

Hirose, S. (1997). Determination of the capacity of a regulating pond in a pipeline irrigation system. Rural and Env. Engrg., 33, 66-78.

Jowitt P.W. \& Germanopoulos, G. (1992). Optimal pump scheduling in water-supply networks. J. Water Resour. Planning and Mgmt., 118(4), 406-422.

JCRMO. (2012). Inventario de regadíos de la JCRMO. Memorias anuales. España: Junta Central de Regantes de la Mancha Oriental. http://www.jcrmo.org/index.php?id=38.

Keller, J., \& Bliesner, R.D. (1990). Sprinkle and trickle irrigation. New York, NY, USA: AVI Book, Van Nostrand Reinholh.

López-Luque, R., Reca, J., \& Roldán, J. (1993). Algoritmo de selección y gestión de tarifas de energía eléctrica en los sistemas de impulsión de agua. Actas de las XI Jornadas Técnicas sobre Riegos, Valladolid, España, (pp 323-328).

Molina, J.M. (2004). Caracterización y modelización de la evaporación en tanques evaporímetros y embalses de riego. Evaluación de los efectos de mallas de sombreo. Tesis doctoral, Universidad Politécnica de Cartagena, España. 207 p.

Moreno, M.A., Medina. D., Ortega, J.F. \& Tarjuelo, J.M. (2012). Optimal design of center pivot systems with water supplied from wells. Agricutural Water Manage, doi:10.1016/j.agwat.2012.01.016.

Moreno, M.A., Planells, P., Ortega, J.F., \& Tarjuelo, J.M. (2007). New methodology to evaluate flow rates in on-demand irrigation networks. Journal of irrigation and drainage engineering (ASCE), 133(4), 298-306.

Nel, D., \& Haarhoff, J. (1996). Sizing municipal water storage tanks with Monte Carlo simulation. J. Water SRT-Aqua, 45(4), 203-212.

Nelder, J.A., \& Mead, R. (1965). A simplex method for function minimization. Comput. Journal., 7(4), 308-313.

Pulido-Calvo, I., Gutiérrez-Estrada, J.C., López-Luque, R., \& Roldán, J. (2006). Regulating reservoirs in pressurized irrigation water supply systems. Journal. Water SRT-Aqua, 55(5), 367-381.

Pulido Calvo I., Gutierrez, J.C., Roldán, J., \& López, R. (2006). Depósitos de regulación en cabeceras de redes hidráulicas a presión. Revista Iberoamericana de Ingeniería Mecánica, 10(2), 81-96.

Pulido-Calvo, I., Roldán, J., López-Luque, R., \& Gutierrez-Estrada, J.C. (2003). Water delivery planning considering irrigation simultaneity. J. Irrig. Drain. Eng., 129 (4), 247255.

Pulido-Calvo, I. \& Gutiérrez-Estrada, J.C. (2011). Selection and operation of pumping stations of water distribution systems. Environmental Research Journal, 5(3), 1-20.

Reca, J., García-Manzano, A., \& Martínez, J. (2014). Optimal pumping scheduling model considering reservoir evaporation. Agricutural Water Manage, 148, 250-257.

Scherer, T., \& Weigel, J. (1993). Planning to irrigate. NDSU extension service. North Dakota State University. http://www.ext.nodak.edu/extpubs/ageng/-irrigate/ae91w.htm. 